\title{
COVID-19 vaccine as a cause for unilateral lymphadenopathy detected by 18 F-FDG PET/CT in a patient affected by melanoma
}

\author{
Mordechai Avner $^{1} \cdot$ Marina Orevi $^{2} \cdot$ Nadia Caplan $^{3} \cdot$ Aron Popovtzer $^{1}$ - Michal Lotem ${ }^{1,4} \cdot$ Jonathan E. Cohen $^{1,4}$ (D)
}

Received: 12 February 2021 / Accepted: 22 February 2021 / Published online: 6 March 2021

(C) The Author(s), under exclusive licence to Springer-Verlag GmbH Germany, part of Springer Nature 2021

A 57-year-old male with BRAF mutant melanoma of the right thigh with lung, inguinal lymph node, and spleen metastases reached complete remission under combined treatment with targeted therapy (dabrafenib and trametinib) and anti-PD-1 (pembrolizumab). 18F-FDG-PET/CT and abdominal MRI from September 2020 showed no active disease.

Two weeks after the most recent dose of pembrolizumab, a routine PET-FDG showed newly enlarged multiple lymph nodes in the left axilla, retro pectoral space, and proximal arm, with high FDG uptake (up to SUV 9.4); no other foci were identified (see Figure). Upon questioning, the patient reported that he had received two doses of the Pfizer COVID-19 vaccine in his left arm, the last of which was given 6 days prior to imaging acquisition.

Axillary lymphadenopathy has been reported in $0.3 \%$ of BNT162b2 mRNA COVID-19 vaccine recipients and was usually resolved within 10 days post-injection [1]. Indeed, FDG-avid lymphadenopathy was described after COVID-

This article is part of the Topical Collection on Image of the month.

Jonathan E. Cohen

cohenjon@hadassah.org.il

1 Sharett Institute of Oncology, Hadassah Medical Center, The Faculty of Medicine, Hebrew University of Jerusalem, Jerusalem, Israel

2 Department of Medical Biophysics and Nuclear Medicine, Hadassah Medical Center, The Faculty of Medicine, Hebrew University of Jerusalem, Jerusalem, Israel

3 Division of Radiology and Medical Imaging, Hadassah Medical Center, The Faculty of Medicine, Hebrew University of Jerusalem, Jerusalem, Israel

4 The Wohl Institute for Translational Medicine, Hadassah Medical Center, Jerusalem, Israel
19 vaccination [2]. Similar findings are also reported after influenza vaccination [3]. In this case, the occurrence of post vaccination lymphadenopathy is described in a melanoma patient receiving immunotherapy. The contribution of immune checkpoint inhibitors targeting the PD-1/PD-L1 axis has not yet been recorded, but the intensity of adenopathy may be attributed, at least in part, to the enhancing effects of immunotherapy. While PD-1 blockade does not appear to affect the severity of COVID-19 infection [4], it significantly enhances the emerging immune response by vaccines such as influenza [5].

With widespread deployment of COVID-19 vaccines and in-line with recent endorsement of vaccination in oncological patients under active anti-tumor treatment, similar findings to those we describe are likely to be encountered widely. It is important to keep this etiology for axillary lymphadenopathy in mind when interpreting PET scans in oncological patients. 

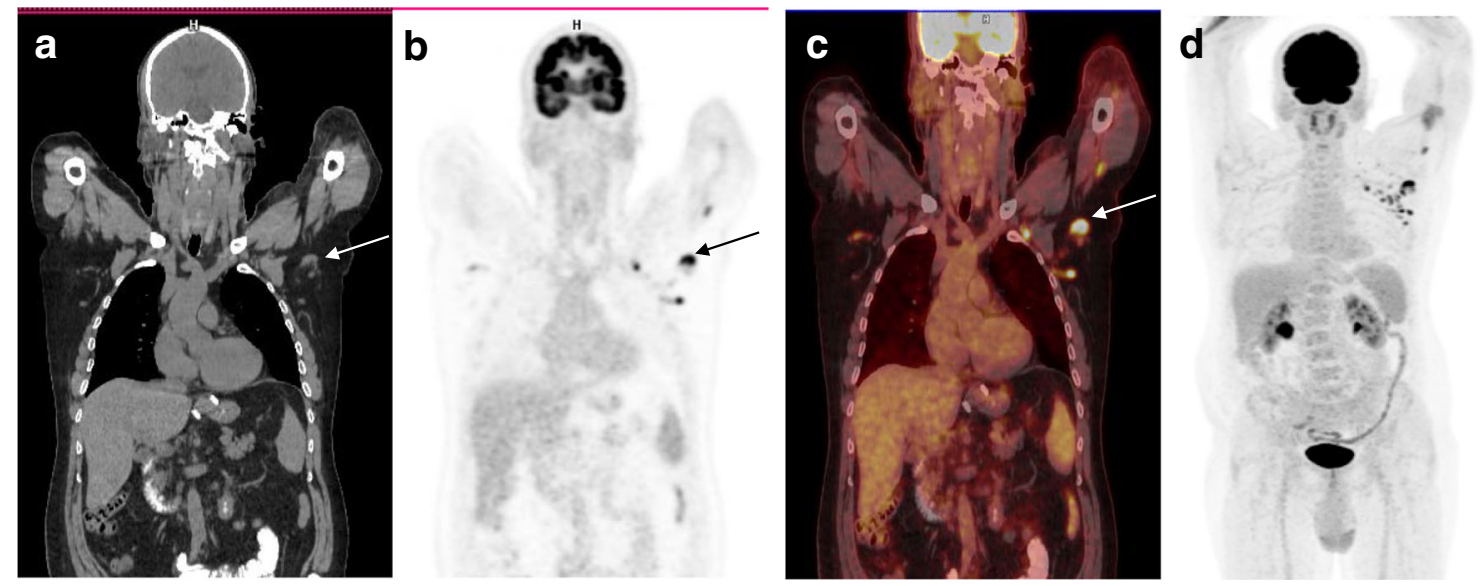

\section{Declarations}

2. Eifer M, Eshet E. Imaging of COVID-19 vaccination at FDG PET/ CT. Images Radiology. 2021. https://doi.org/10.1148/radiol. 2020210030.

Conflict of interest The authors have no relevant financial or nonfinancial interests to disclose.

The patient gave written consent regarding publishing their data and images.

\section{References}

1. Polack FP, Thomas SJ, Kitchin N, Absalon J, Gurtman A, Lockhart S, et al. Safety and efficacy of the BNT162b2 mRNA Covid-19 vaccine. NEJM. 2020;383(27):2603-15.

3. Shirone N, Shinkai T, Yamane T, Uto F, Yoshimura H, Tamai H, et al. Axillary lymph node accumulation on FDG-PET/CT after influenza vaccination. Ann Nucl Med. 2012;26(3):248-52.

4. Luo J, Rizvi H, Egger JV, Preeshagul IR, Wolchok JD, Hellmann MD. Impact of PD-1 blockade on severity of COVID-19 in patients with lung cancers. Cancer Discov. 2020;10(8):1121-8.

5. Kang CK, Kim HR, Song KH, Keam B, Choi SJ, Choe PG, et al. Cell-mediated immunogenicity of influenza vaccination in patients with cancer receiving immune checkpoint inhibitors. J Infect Dis. 2020;222(11):1902-9.

Publisher's note Springer Nature remains neutral with regard to jurisdictional claims in published maps and institutional affiliations. 